\title{
Iodine status in breastfeeding women and their infants in New Zealand
}

\section{Abstract}

Iodine is an essential micronutrient in thyroid hormone synthesis and physical and mental development. Iodine deficiency has historically been a problem in New Zealand. Two government initiatives were introduced: 1) mandatory fortification of all bread (except organic) with iodised salt (2009); 2) provision of a subsidised iodine supplement for all pregnant and breastfeeding women (2010). Since these initiatives, the majority of adults and children have achieved adequate iodine status. However, iodine status among breastfeeding women and their infants is unknown. This observational follow-up cohort study recruited 87 breastfeeding women, aged 16 years and over, with a healthy term singleton infant, in the Manawatu region, New Zealand. Women were recruited at 3 months postpartum; and followed up at 6 and 12 months postpartum. A maternal questionnaire investigated supplement use before and after delivery, iodine nutritional knowledge, and demographic information. Maternal spot urine, breastmilk, and infant spot urine samples were collected and iodine concentrations determined using inductively-coupled plasma mass spectrometry. Iodine-containing supplement use was recorded in the 24 hours before biological sample collection. Shapiro-Wilk test was used to check the normality of the data. Iodine data were analysed using non-parametric Mann Whitney U test (2-tailed).Thirty-five (40\%) women used iodine-containing supplements during breastfeeding. Median urine iodine concentration (UIC) was $82(46,157) \mu \mathrm{g} / \mathrm{L}$, below the WHO cut-off value of $100 \mu \mathrm{g} / \mathrm{L}$ for breastfeeding women. Median infant UIC was $115(69,182) \mu \mathrm{g} / \mathrm{L}$, above the WHO cut-off value of $100 \mu \mathrm{g} / \mathrm{L}$. Median maternal UIC was significantly higher for supplement users than non-users $(111(44,240)$ vs $62(42$, 102) $\mu \mathrm{g} / \mathrm{L} ; \mathrm{p}=0.021]$. Median infant UIC was significantly higher for supplement users than non-users $(150(97,193)$ vs $86(53$, 171) $\mu \mathrm{g} / \mathrm{L} ; \mathrm{p}=0.045]$. Median breastmilk iodine concentration $(\mathrm{BMIC})$ was $69(52,119) \mu \mathrm{g} / \mathrm{L}$, lower than recommended $(75 \mu \mathrm{g} / \mathrm{L})$. Median BMIC was significantly higher for supplement users than non-users $[83(67,168)$ vs $62(48,82) \mu \mathrm{g} / \mathrm{L} ; \mathrm{p}=0.000]$. Overall, iodine status within this sample of breastfeeding women remains inadequate at 3 months postpartum. Users of iodine containing supplements and their infants achieved recommended iodine UIC concentrations; however, non-users and their infants had inadequate status. Despite recommendations that all breastfeeding women use iodine supplements, supplement use was low (only $40 \%$ ). Further work is require to investigate if current iodine status affects thyroid function. Alternative strategies are also required to ensure breastfeeding women and their infants have adequate iodine.

\section{Conflict of Interest}

There is no conflict of interest 